\title{
Rhino-Cerebral Mucormycosis with Otologic Involvement
}

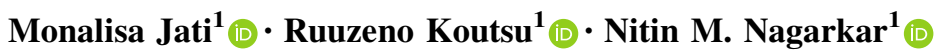

Received: 15 July 2021/ Accepted: 21 November 2021/Published online: 8 January 2022

(C) Association of Otolaryngologists of India 2021

\begin{abstract}
Mucormycosis is an opportunistic invasive fungal infection, recently been detected in Covid-19 due to several underlying conditions. Otologic involvement in such patients though exceptional, should not be simply ignored even when presented without any clinical signs or symptoms. This report manifests the need of evaluating otologic involvement in post-covid mucormycosis.
\end{abstract}

Keywords Mucormycosis - Hearing loss ·

Otologic involvement · Audiological investigation

\section{Introduction}

Covid-19 is still wreaking havoc in every country, including India, after more than a year. While the nation was attempting to maintain stability in the face of the current pandemic, the mutant virus began to cause new symptoms, with a new threat in the form of mucormycosis. In the second wave of Covid-19, mucormycosis infections spiked [1].

Mucormycosis is an aggressive fungal opportunistic infection resulting in acute to extremely severe symptoms. Otologic involvement in such cases is rare, therefore any

Monalisa Jati

monalisajati@gmail.com

Ruuzeno Koutsu

ruuzenokuotsu@gmail.com

Nitin M. Nagarkar

director@aiimsraipur.edu.in

1 Department of Otolaryngology and Head and Neck Surgery, All India Institute of Medical Sciences, Raipur, Chhattisgarh 492099, India such involvement should be recognised and treated right away before it has any significant consequences.

Here we present a case of moderately severe mixed hearing loss with eustachian tube dysfunction in a post covid-19 mucormycosis patient, which was caused by the organism.

\section{Case Report}

A 65-year old female presented to our hospital with a ten days history of swelling, pain, weakness on the right side of her face, and blurring of vision in the right eye after being exposed to covid-19. She was a longstanding diabetic (>3 years) on oral antihypoglycemic tablets. The physical examination revealed partial right proptosis with normal neurological examination except right abducens nerve palsy and grade-III peripheral facial palsy. The laboratory reports tested positive for fungal element, and a positive fungal culture report was suggestive of Mucormycosis.

An otoscopic examination was performed revealing normal tympanic membrane and external auditory canal. Then a detailed audiological investigation was done. Pure tone audiometry (PTA) demonstrated, moderately severe mixed hearing loss for the right ear, and normal hearing with high frequency loss for the left ear (Fig. 1). Tympanometry showed C-type and A- type tympanograms for right and left ear indicating eustachian-tube dysfunction and normal middle ear status for right and left ear respectively.

A contrast- enhanced CT and magnetic resonance imaging (MRI) of head was performed revealing soft tissue thickening of the mucosa of the right ethmoid, maxillary, and sphenoid sinuses, with extension into the naso-pharyngeal mucosal space and right pterygoid muscle, as well 
Fig. 1 The pure tone audiometry results at the time of routine clinical examination: RE: Moderately severe Mixed hearing loss. LE: Hearing sensitivity within Normal Limits with high frequency loss

\section{PURE TONE AUDIOGRAM}

RICHT

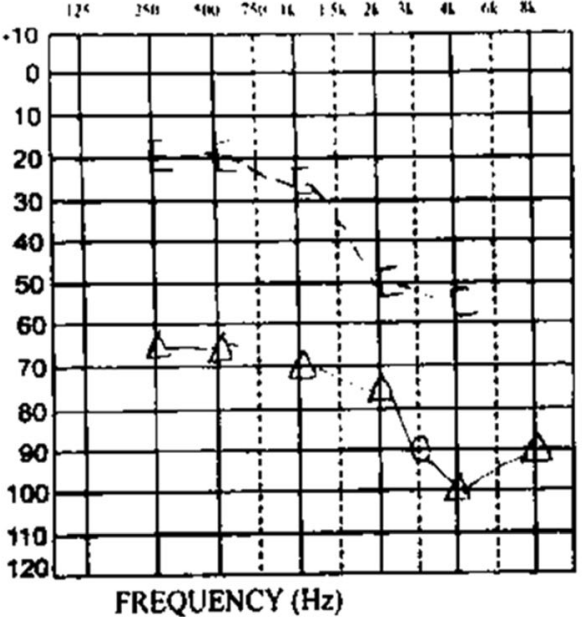

LEFT

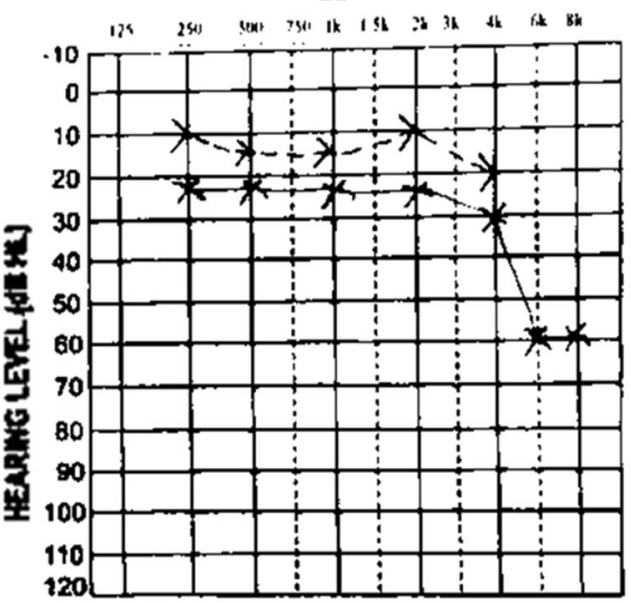

FREQUENCY $(\mathrm{Hz})$ as spread via the right eustachian-tube to the right middle ear and right mastoid air cells. It also revealed an irregular peripheral enhancing lesion in the inferior right temporal lobe and associated mild perifocal edema without intraorbital extension.

Histopathological examination revealed broad non-septate hyphae accompanied by neutrophils and lymphocytes with necrotic foci on PAS and Grocott staining (Fig. 2), suggestive of a mucor fungus, and the patient was pathologically diagnosed with invasive mucormycosis.

The patient was diagnosed with rhino-cerebral mucormycosis and was started on intravenous amphotericin B $(0.5 \mathrm{mg} / \mathrm{kg} /$ day $)$ right away, and surgical debridement was advised as a life-saving approach. An extensive surgical debridement was performed after 5 days of admission by a multidisciplinary team of Otolaryngologists and Neurosurgeons. Otolaryngologists performed sinus debridement, while neurosurgeons removed necrotic tissue from the brain. The patient was extubated after surgery and admitted to the critical care unit (CCU) for further management.

\section{Discussion}

The otologic involvement in mucormycosis is not well anticipated, due to its rarity, and lack of distinct clinical symptoms. In the present case, even before scheduling her for any imaging studies, we hypothesized that her hearing loss was due to the progression of mucormycosis affecting the auditory system.

Mucormycosis of the ear is uncommon, and when it appears, it tends to affect the temporal bone and external ear $[2,3]$. We came across a case study of auricular mucormycosis, in which chronic otitis media with otalgia, otorrhea, and hearing loss had been reported whereas another case study reported narrowed and inflammatory external auditory canal with tympanic membrane perforation $[4,5]$. One of the possible paths postulated for otologic involvement, is the entrance of the organism into the middle ear, either via the eustachian-tube or a perforated tympanic membrane [6].

In the present case, there was neither any prior history of hearing loss nor any clinical otologic symptoms when the patient was brought to our hospital. Therefore we concluded mucormycosis was the sole cause of hearing loss in our case. The fungal infection spread to the middle ear via the eustachian tube, was indicated by the audiological testing and, later, was confirmed by the radiological investigation. We further consider that the ototoxicity of the covid-19 therapy impacted high frequencies, damaging the inner ear and that this was the reason for the mixed component of hearing loss in this patient.

The aim of this study is to highlight that, in the past, when the auditory system was associated with mucormycosis, some or other otological symptoms were observed, but none were seen in our case, making this case study exceptional [2-5]. Otologic mucormycosis is difficult to diagnose because of its rarity, lack of awareness, and lack of distinct clinical signs and symptoms. Therefore this case study establishes that even though mucormycosis patients present no visible otologic symptoms, the likelihood of auricular involvement should be addressed and investigated by professionals. If not detected early the infection may spread to the inner ear and ultimately to the brain via the temporal bone, culminating in mortality.

In a recent review, a variety of tests for mucormycosis patients were described, however none of the studies 


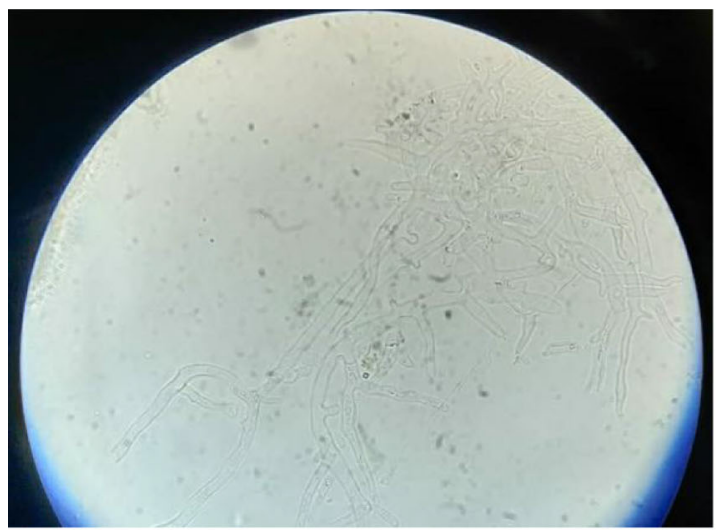

A

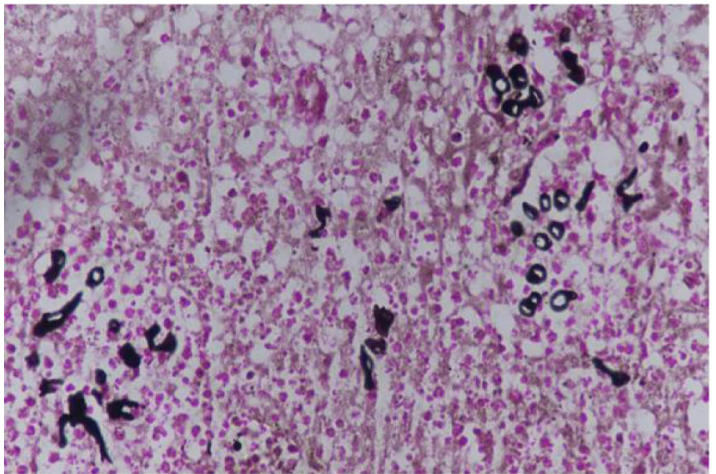

C

Fig. 2 Histopathology slides. a $\mathrm{KOH}$ mount broad aseptate hyphae with branching. b Histopathology smear showing broad non-septate hyphae of mucor accompanied by neutrophils and lymphocytes with

included audiological investigation [7]. Therefore we propose a comprehensive audiological assessment be performed as a part of routine investigations in mucormycosis patients.

\section{Conclusion}

The mucormycosis is an opportunistic infection in post covid-19, causing acute to extremely severe symptoms. Due to the rarity of otologic involvement in such situations, access to it is limited. Any delay in recognising and resolving such problems might raise the chances of losing the battle against mucormycosis. In this regard, there is a stronger need for population-based research that may demonstrate clinical symptoms and treatment of hearing loss caused by mucormycosis, which can then be transferred into everyday practise in this pandemic, enhancing the best practice. Till then more case studies like these, which are critical in raising awareness of the likelihood of otologic involvement in post-covid-19 mucormycosis need

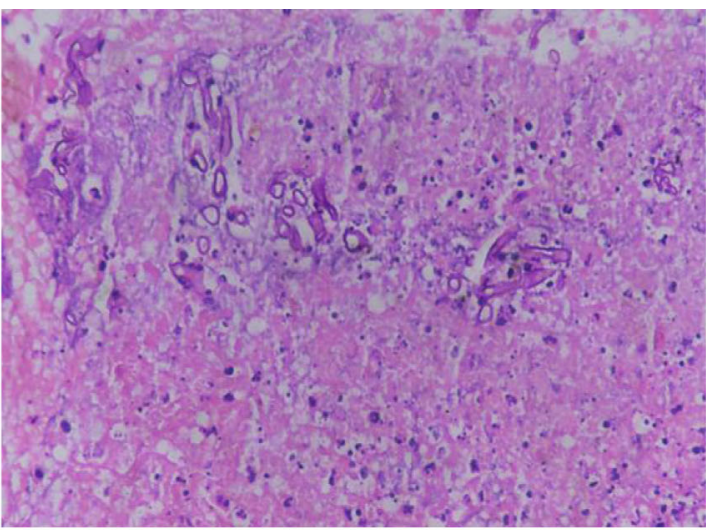

B

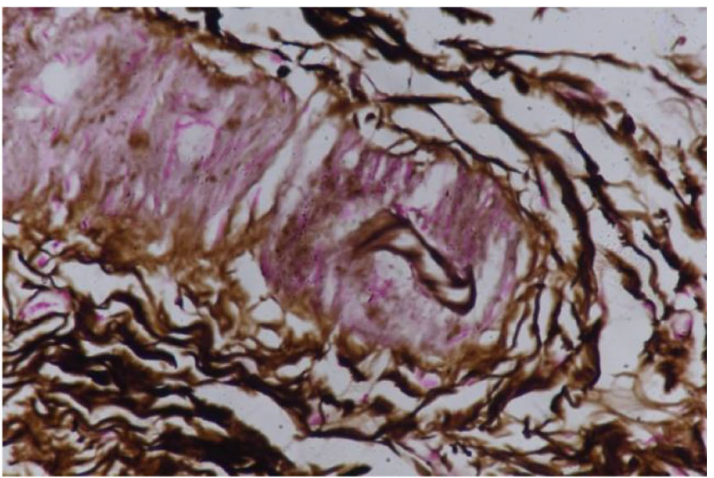

D

necrotic foci. c, d Grocott- Gomori methanamine-silver (GMS) stain highlighting fungal elements

to be put on public platform for the references of professionals.

Funding None.

\section{Declarations}

Conflict of interest The authors declare that they have no conflict of interest.

\section{References}

1. John TM, Jacob CN, Kontoyiannis DP (2021) When uncontrolled diabetes mellitus and severe COVID-19 converge: the perfect storm for mucormycosis. J Fungi 7(4):298. https://doi.org/10.3390/jof7040298

2. Tuzcu A, Bahceci M, Celen MK, Kilinc N, Ozmen S (2006) Necrotizing (malignant) Otitis externa: an unusual localization of mucormycosis. Indian J Med Microbiol 24(4):289-291. https://doi.org/10.4103/0255-0857.29390

3. Biniyam K, Bhat V, Bhandary SK, Aroor R (2014) Asymptomatic mucormycosis of middle ear: an incidental finding during 
tympanoplasty. Indian J Otol 20(2):83. https://doi.org/10.4103/0971-7749.131875

4. Bellazreg F, Hattab Z, Meksi S, Mansouri S, Hachfi W, Kaabia N, Ben Said M, Letaief A (2014) Outcome of mucormycosis after treatment: report of five cases. New Microb New Infect 6:49-52. https://doi.org/10.1016/j.nmni.2014.12.002

5. Kermani W, Bouttay R, Belcadhi M, Zaghouani H, Ali MB, Abdelkéfi M (2016) ENT mucormycosis. Report of 4 cases. Eur Ann Otorhinolaryngol Head Neck Dis 133(2):83-86. https://doi.org/10.1016/j.anorl.2015.08.027

6. Hazarika P, Zachariah J, Victor J, John M, Devi C, Abraham P (2012) Mucormycosis of the middle ear: a case report with review of literature. Indian J Otolaryngol Head Neck Surg 64(1):90-94. https://doi.org/10.1007/s12070-011-0156-3

7. Skiada A, Pavleas I, Drogari-Apiranthitou M (2020) Epidemiology and diagnosis of mucormycosis: an update. J Fungi 6(4):265. https://doi.org/10.3390/jof6040265

Publisher's Note Springer Nature remains neutral with regard to jurisdictional claims in published maps and institutional affiliations. 